\title{
Mesures d'équilibre sur un reseau
}

\author{
F. Ledrappier * \\ Laboratoire de Probabilités, Paris, France
}

Received March 15, 1973

\begin{abstract}
Some one-dimensional lattice systems with infinite range interactions define a Bernoulli shift.
\end{abstract}

\section{Introduction et résultats}

Soit $\Phi$ une fonction réelle sur les parties finies de $Z^{v}$ vérifiant les propriétés suivantes:

a) $\Phi(\emptyset)=0$,

b) $\Phi(X+a)=\Phi(X)$,

c) $\sum_{x>0}|\Phi(X)|<+\infty$.

Une telle fonction est appelée une interaction et peut représenter un système de mécanique statistique (cf. [4]). L'ensemble de ces interactions forme un Banach pour la norme $\|\Phi\|$ définie par c). On pose $U(X)=\sum_{Y \subset X} \Phi(X)$, pour $X$ partie finie de $Z^{v}$. On a $U(X) \leqq N(X)\|\Phi\|$, où $N(X)$ désigne le cardinal de $X$. Soit $K$ l'espace produit $\{0,1\}^{Z^{v}}$ muni de la $\sigma$-algèbre $\mathscr{A}$ engendrée par les applications coordonnées. Pour toute partie $\Delta$ de $Z^{v}$, on notera $P_{\Delta}$ la $\sigma$-algèbre engendrée par les applications coordonnées qui sont dans $\Delta$. Soit $G$ le groupe d'applications bimesurables de $K$ sur lui-même définies par les translations des coordonnées.

Pour tout cylindre fini ou infini $A, A=\left\{x, x=x_{i}, i \in Z^{v}, x \in K\right.$, $\left.x_{i}=0 i \in \Delta_{0}, x_{i}=1 i \in \Delta_{1}\right\}$ on note $\hat{A}$ la partie $\Delta_{1}$ de $Z^{v}$.

Une probabilité $\mu$ sur $K$ est dite une mesure d'équilibre pour l'interaction $\Phi$ si on a la relation suivante: pour toute partie finie $\Delta$ de $Z^{\nu}$, pour tout atome $\alpha$ de $P_{\Delta}, E_{\mu}^{P_{\Delta^{c}}}\left(1_{\alpha}\right)(y)=f(\alpha, y) E_{\mu}^{P^{\Delta^{c}}}\left(1_{\alpha_{0}}\right)(y)$ pour $\mu$-presque tout $\tilde{y}$ appartenant à $\left(K, P_{\Delta^{c}}\right)$, où $\alpha_{0}=\left\{\omega ; \omega \in K, \omega_{j}=0 \forall j \in \Delta\right\}, f(\alpha, y)$ $=\exp (-U(\hat{\alpha})-W(\hat{\alpha}, \hat{y}))$ avec pour $X_{1}, X_{2}$ dans $Z, W\left(X_{1}, X_{2}\right)=\sum_{\substack{Y \subset X_{1} \cup X_{2} \\ Y \cap X_{i} \neq \emptyset}} \Phi(Y)$.

Nous allons d'abord donner les propriétés qui résultent de cette définition, en particulier dans le cas où il n'existe qu'une seule mesure d'équilibre.

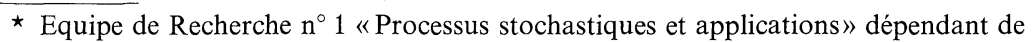
la Section $\mathrm{n}^{\circ} 1$ «Mathématiques, Informatique» associée au C.N.R.S. 
Nous étudierons ensuite dans le cas où $v=1$ un espace particulier d'interactions $B_{1}$ pour lequel on peut montrer:

Théorème 1. Si $\Phi$ appartient à $B_{1}$, alors il n'existe qu'une mesure d'équilibre et le système $(K, \mu, G)$ est un schéma de Bernoulli.

Les résultats et les démonstrations s'étendent sans difficultés au cas des mesures sur $\{0,1, \ldots, N-1\}^{Z^{v}}$.

\section{Existence de mesures d'équilibre et propriétés}

On va noter

$$
\begin{gathered}
\Delta_{n}=\left\{a, a \in Z^{v}, a=\left(a_{1}, a_{2}, \ldots, a_{v}\right)\left|a_{i}\right| \leqq n \forall i\right\}, \\
y_{n}(x)=\left\{\omega, \omega \in K \quad \omega_{i}=x_{i} \forall i \notin \Delta_{n}\right\}, \\
Z_{n}(x) \text { la fonction }\left(\sum_{\alpha \in P_{\Delta_{n}}} f\left(\alpha, y_{n}(x)\right)^{-1} .\right.
\end{gathered}
$$

On désigne par $\phi_{n}$ l'application de $\mathscr{L}(K)$ dans $\mathscr{L}(K)$, ensemble des fonctions $\mathscr{A}$-mesurables sur $K$, définie par:

$$
\phi_{n} h(x)=Z_{n}(x) \sum_{\alpha \in P_{\Delta_{n}}} f\left(\alpha, y_{n}(x)\right) h\left(\alpha \cap y_{n}(x)\right) .
$$

Les $\phi_{n}$ sont des opérateurs positifs de $\mathscr{L}(K)$ dans $\mathscr{L}(K)$ et $\phi_{n} 1=1$.

Si $K$ est muni de la topologie produit des topologies discrètes sur $\{0,1\}$, alors $K$ est compact et les $\phi_{n}$ sont des opérateurs positifs continus de $C(K)$ dans $C(K)$, espace des fonctions réelles continues muni de la norme uniforme.

On note $\phi_{n}^{*}$ l'opérateur transposé de $\phi_{n}$, de $M(K)$ dans $M(K)$, ensemble des mesures de Radon sur $K ; \phi_{n}^{*}$ est un opérateur positif et conserve l'ensemble des probabilités de Radon sur $K$, qui est faiblement compact. On a:

Proposition 1. Soit $v$ une probabilité de Radon sur $K, \mu$ un point d'adhérence faible de $\left\{\phi_{n}^{*} v, n>0\right\}$. Alors $\mu$ est une mesure d'équilibre.

Démonstration. Soit $\Delta$ un ensemble fini de $Z^{v}$ et $\alpha$ un atome de $P_{\Delta}$. Soit $n$ assez grand pour que $\Delta_{n}$ contienne $\Delta$ et $\beta$ un atome de $P_{\Delta_{n}}$. On va calculer $\phi_{m} 1_{\alpha \cap \beta}$ pour $m$ plus grand que $n$.

$$
\begin{aligned}
\phi_{m} 1_{\alpha \cap \beta}= & Z_{m}(x) \sum_{\gamma \in P_{\Delta_{m}} \backslash \Delta_{n}} f\left(\alpha \cap \beta \cap \gamma, y_{m}(x)\right) \quad \text { ou en décomposant } \\
& f\left(\alpha \cap \beta \cap \gamma, y_{m}(x)\right): \\
= & Z_{m}(x) \sum_{\gamma \in P_{\Delta_{m}} \backslash \Delta_{n}} \exp -(U(\alpha)+U(\beta)+U(\gamma)+W(\alpha, \beta \cap \gamma)+W(\beta, \gamma) \\
& +W\left(\alpha \cap \beta \cap \gamma, y_{m}(x)\right) .
\end{aligned}
$$


En choisissant $n$ assez grand, pour tout $\varepsilon$ on peut assurer que:

$|W(\alpha, \beta \cap \gamma)-W(\alpha, \beta)|<\varepsilon / 2 \quad\left|W\left(\alpha \cap \beta \cap \gamma, y_{m}(x)\right)-W\left(\beta \cap \gamma, y_{m}(x)\right)\right|<\varepsilon / 2$ uniformément en $\alpha \in \Delta, \beta \in P_{\Delta_{n} \backslash \Delta}, \gamma \in P_{\Delta_{m} \backslash \Delta_{n}}$ et $x$.

On a alors, où $A_{m}(\beta, x)$ ne dépend pas de $\alpha$ :

$$
\begin{gathered}
\exp -(U(\alpha)+W(\alpha, \beta)+\varepsilon) A_{m}(\beta, x) \\
\leqq \phi_{m} 1_{\alpha \cap \beta} \leqq \exp -(U(\alpha)+W(\alpha, \beta)-\varepsilon) A_{m}(\beta, x)
\end{gathered}
$$

en sommant en $\alpha$ on obtient la même estimation pour $\phi_{m} 1_{\beta}$. En intégrant en $v$, on obtient:

$$
\frac{e^{-\varepsilon} f(\alpha, \beta)}{\sum_{\alpha \in P_{\Delta}} e^{\varepsilon} f(\alpha, \beta)} \leqq \phi_{m}^{*} v(\alpha / \beta) \leqq \frac{e^{\varepsilon} f(\alpha, \beta)}{\sum_{\alpha \in P_{\Delta}} e^{-\varepsilon} f(\alpha, \beta)} .
$$

Soit $\mu$ un point d'adhérence faible de la suite $\phi_{m}^{*} v$, comme $1_{\alpha \cap \beta}$ et $1_{\beta}$ sont continues, on a $\mu(\alpha / \beta)$ compris entre les mêmes bornes. Ceci étant vrai pour tout $\beta$ de $P_{\Delta_{n} \backslash \Delta}$, on a également $E_{\mu}^{\Delta_{n} \backslash \Delta} 1_{\alpha}(\beta)$ compris entre les mêmes bornes.

Quand $n$ tend vers l'infini, $E_{\mu}^{P_{\Delta_{n} \backslash \Delta}} 1_{\alpha}$ converge $\mu$-presque sûrement vers $E_{\mu}^{P_{\Delta_{c}}} 1_{\alpha}$, alors que les bornes convergent simplement toutes deux vers $\frac{f(\alpha, x)}{\sum_{\alpha \in P \Delta} f(\alpha, x)}$, qui est bien une fonction $P_{\Delta^{c}}$ mesurable. On a bien la relation d'équilibre en remarquant que $f\left(\alpha_{0}, x\right)=1$.

Remarque. La démonstration habituelle de l'existence est faite avec $\nu=\delta_{\varrho}$, ò̀ $\varrho=\left\{\varrho_{i} i \in Z^{v}, \varrho_{i}=0\right.$ pour tout $\left.i\right\}$.

Proposition 2. Il n'existe qu'une seule mesure d'équilibre pour $\Phi$ si et seulement si pour tout $f$ de $C(K), \phi_{n} f$ converge uniformément vers une constante $\tilde{f}$.

Démonstration. Supposons d'abord que pour toute fonction continue $\phi_{n} f$ converge uniformément vers une constante $\tilde{f}$. Soit $\mu$ une mesure d'équilibre, $\alpha$ un cylindre fini. Comme dès que $\alpha$ est $P_{\Delta_{n}}$ mesurable, $\phi_{n} 1_{\alpha}=E_{\mu}^{P \Delta_{n}^{c}} 1_{\alpha} \mu$-presque sûrement, dire que $\phi_{n} f$ converge uniformément vers une constante permet de dire que les $E_{\mu}^{P \Delta_{n}^{c}} 1_{\alpha}$ convergent en norme \|\|$_{\infty}$ vers cette constante $\tilde{1}_{\alpha}$. On a donc: $\tilde{1}_{\alpha}={E_{\mu}^{n}}^{\wedge P_{\Delta_{n}^{c}}} 1_{\alpha}$. En intégrant en $\mu$, $\mu(\alpha)=\tilde{1}_{\alpha}$.

D'autre part, s'il existe une fonction $f$ telle que les $\phi_{n} f$ ne convergent pas uniformément vers une constante, on peut écrire:

$\exists \varepsilon>0, \exists n_{k}, \exists x_{k}, y_{k}$ de $K$, tels que $\left|\phi_{n_{k}} f\left(x_{k}\right)-\phi_{n_{k}} f\left(y_{k}\right)\right|>\varepsilon$. 
Si $n_{k_{i}}$ est une sous-suite de la suite $n_{k}$ telle que $\phi_{n_{k_{i}}} \delta_{x_{k_{i}}}$ et $\phi_{n_{k_{i}}} \delta_{y_{k_{i}}}$ convergent faiblement vers des mesures $\mu_{1}$ et $\mu_{2}$, une démonstration analogue à celle de la proposition 1 montre que $\mu_{1}$ et $\mu_{2}$ sont des mesures d'équilibre, elles sont différentes car $\left|\mu_{1}(f)-\mu_{2}(f)\right|>\varepsilon$.

Proposition 3. Si une interaction n'admet qu'une seule mesure d'équilibre, le système $(K, \mu ; G)$ est d'entropie complètement positive.

Démonstration. D'abord $\mu$ est $G$-invariante, car d'après l'équation d'équilibre $g \mu$ est également une mesure d'équilibre pour $\Phi$.

Montrons ensuite que $\bigwedge_{n} P_{\Delta_{n}^{c}}$ est triviale. Si en effet la $\sigma$-algèbre $\bigwedge_{n} P_{\Delta_{n}^{c}}$ n'est pas triviale, on peut trouver une fonction $f$ positive, d'intégrale un, non constante et $\bigwedge_{n} P_{\Delta_{n}^{c}}$ mesurable. Alors il est clair que $f \mu$ satisfait aussi l'équation d'équilibre; $f \mu$ est alors une autre mesure d'équilibre pour $\Phi$. Si $\bigwedge_{n} P_{\Delta_{n}^{c}}$ est triviale, alors le plus grand facteur d'entropie nulle $\Pi(P)$ est trivial (cf. [1]); on dit alors que le système est d'entropie complètement positive.

\section{Démonstration du théorème 1}

Dans le cas où $v=1$, on va considérer les $\Phi$ appartenant à $B_{1}$, c'est à dire tels que:

$$
\|\Phi\|_{1}=\sum_{\substack{X \supset 0 \\ X \subset N}} 1(X) \Phi(X)<+\infty \text {, où } 1(X) \text { désigne le diamètre de } X .
$$

On va utiliser la construction précédente, mais avec de nouveaux opérateurs $\phi_{n}^{\prime}$ qui se comportent de la même manière que les $\phi_{n}$.

On dira qu'un point $x^{\prime}$ est $n$-antérieur à $x\left(x^{\prime}\right.$ appartient à $\left.n(x)\right)$ si:

$$
x_{i}^{\prime}=x_{i+n} \text { pour } i \leqq-n, \quad x_{i}^{\prime}=x_{i-n} \text { pour } i>n .
$$

On note $Z_{n}^{\prime}(x)=\left(\sum_{x^{\prime} \in n(x)} f\left(\alpha_{n}\left(x^{\prime}\right), y_{n}\left(x^{\prime}\right)\right)\right)^{-1}$ où maintenant

$$
\begin{aligned}
& \alpha_{n}(x)=\left\{\omega ; \omega \in K, \omega_{i}=x_{i}-n<i \leqq n\right\} . \\
& \left.\left.y_{n}(x)=\left\{\omega ; \omega \in K, \omega_{i}=x_{i} i \notin\right]-n, n\right]\right\} .
\end{aligned}
$$

On définit alors $\phi_{n}^{\prime} h(x)=Z_{n}^{\prime}(x) \sum_{x^{\prime} \in n(x)} f\left(\alpha_{n}\left(x^{\prime}\right), y_{n}\left(x^{\prime}\right)\right) h\left(x^{\prime}\right)$.

Les $\phi_{n}^{\prime}$ ont les mêmes propriétés que les $\phi_{n}$. En particulier, les $\phi_{n} 1_{\alpha}$ convergent uniformément si et seulement si les $\phi_{n} 1_{\alpha}$ convergent uniformément pour $\alpha$ cylindre fini. Mais les $\phi_{n}^{\prime}$ ont maintenant une propriété de composition: 
Proposition 4. Soient $m \geqq n$ deux entiers. Il existe des fonctions continues $\varrho_{n, m}(x)$ telles que, pour tout $h$ de $C(K)$ :

$$
\phi_{m}^{\prime} h(x)=\frac{\sum_{x^{\prime} \in m-n(x)} \varrho_{n, m}\left(x^{\prime}\right) \phi_{n}^{\prime} h\left(x^{\prime}\right)}{\sum_{x^{\prime} \in m-n(x)} \varrho_{n, m}\left(x^{\prime}\right)} .
$$

Démonstration. Il suffit de le montrer pour $n=m-1$. On a d'abord la formule:

$$
U\left(X_{1}, X_{2}\right)+W\left(X_{1} X_{2}, X_{3}\right)=U\left(X_{1}\right)+U\left(X_{2}\right)+W\left(X_{2}, X_{3}\right)+W\left(X_{1}, X_{2} X_{3}\right)
$$

qui est facile à vérifier d'après la définition de $U$ et de $W$.

Soit alors $x$ appartenant à $K, h$ à $C(K)$

$$
\phi_{n} h(x)=\frac{\sum_{\hat{x} \in n(x)} f\left(\alpha_{n}(\hat{x}), y_{n}(\hat{x})\right) h(\hat{x})}{\sum_{\hat{x} \in n(x)} f\left(\alpha_{n}(\hat{x}), y_{n}(\hat{x})\right)} .
$$

Si on écrit $\hat{x}$ comme un point $(n-1)$ antérieur à $x^{\prime}$, où $x^{\prime}$ est lui-même 1 -antérieur à $x$, en notant $\beta$ la réunion des points de $\{-n+1\} \cup\{n\}$ où $\hat{x}_{i}=1$, on obtient

$$
f\left(\alpha_{n}(\hat{x}), y_{n}(\hat{x})\right)=\exp -\left(U(\beta)+W\left(\beta, \widehat{y_{n}(\hat{x})}\right)\right) f\left(\alpha_{n-1}(\hat{x}), y_{n-1(\hat{x})}\right) .
$$

Donc si on regroupe tous les $\hat{x}$ qui ont le même $\beta$ (c'est à'dire $(n-1)$ antérieurs au même $\left.x^{\prime}\right), \phi_{n}^{\prime} h(x)$ s'écrit:

$$
\phi_{n} h(x)=\frac{\sum_{\beta} \exp -\left(U(\beta)+W\left(\beta, \widehat{y_{n}(\hat{x})}\right)\right) \sum_{\hat{x}} f\left(\alpha_{n-1}(\hat{x}), y_{n-1}(\hat{x})\right) h(\hat{x})}{\sum_{\beta} \exp -\left(U(\beta)+W\left(\beta, \widehat{y_{n}(\hat{x})}\right)\right) \sum_{\hat{x}} f\left(\alpha_{n-1}(\hat{x}), y_{n-1}(\hat{x})\right)} .
$$

Mais

$$
\sum_{\hat{x} \in n-1\left(x^{\prime}\right)} f\left(\alpha_{n-1}(\hat{x}), y_{n-1}(\hat{x})\right) h(\hat{x}) \quad \text { est } \quad \phi_{n-1}^{\prime} h\left(x^{\prime}\right) \times Z_{n-1}^{\prime}\left(x^{\prime}\right)^{-1} \text {. }
$$

En posant $\varrho_{n-1, n}\left(x^{\prime}\right)=\exp -\left(U(\beta)+W\left(\beta, \widehat{y_{n}(\hat{x})}\right)\right) Z_{n-1}^{\prime}\left(x^{\prime}\right)^{-1}$, on a la formule annoncée.

Corollaire. $\inf _{x} \phi_{n}^{\prime} h(x)$ croit en $n$ vers $m(h), \sup _{x} \phi_{n}^{\prime} h(x)$ décroit vers $M(h)$.

Pour $\Phi \in B_{1}$, on uțilisera le fait qu'on peut définir alors

$$
W\left(x_{0}, x_{1}\right)=\sum_{X \subset \hat{x}_{0} \cup \hat{x}_{1}, X \cap \hat{x}_{1} \neq \emptyset} \Phi(X),
$$

où $x_{0}$ (respectivement $x_{1}$ ) est un cylindre infini atomique pour $P_{(-\infty, 0]}$ $\left(P_{[1,+\infty)}\right)$. On a même $\left|W\left(x_{0}^{m}, x_{1}^{m}\right)-W\left(x_{0}, x_{1}\right)\right| \leqq k_{m}$ qui décroit uni- 
formément en $x_{0}$ et $x_{1}$ vers 0 , si $x_{0}^{m}$ (respectivement $x_{1}^{m}$ ) est le cylindre dont les coordonnées sont les $m$ premières de $x_{0}$ (respectivement $x_{1}$ ).

La proposition suivante remontre des résultats de [5]:

Proposition 5. Si $\Phi \in B_{1}$ il n'existe qu'une mesure d'équilibre.

Démonstration (cf. [3]). Soit $\alpha$ un cylindre fini, on va montrer que $m\left(1_{\alpha}\right)=M\left(1_{\alpha}\right)$ cela suffit bien pour entraîner que les $\phi_{n}^{\prime} 1_{\alpha}$ convergent uniformément vers une constante

i) Montrons que les $\phi_{n}^{\prime} 1_{\alpha}$ sont équi-continues: si $x$ et $x^{\prime}$ sont deux points de $K$ dont les coordonnées entre $-m$ et $m$ coïncident et si $\alpha_{n}$ est un atome de $P_{]-n, n]}$, on $a$ :

$e^{-2 k_{m}} \leqq \frac{f\left(\alpha_{n}, y_{n}(\hat{x})\right)}{f\left(\alpha_{n}, y_{n}\left(\hat{x}^{\prime}\right)\right)} \leqq e^{2 k_{m}}$, où $\hat{x}$ est un point quelconque $n$-antérieur à $x$, alors dès que $n$ est assez grand pour que $\alpha$ soit $P_{]-n, n]}$ mesurable, on a:

$$
\left|\phi_{n}^{\prime} 1_{\alpha}(x)-\phi_{n}^{\prime} 1_{\alpha}\left(x^{\prime}\right)\right| \leqq\left(e^{2 k_{m}}-1\right) \sup _{n} \sup _{x} \phi_{n}^{\prime} 1_{\alpha}(x) . \quad \text { C.Q.F.D. }
$$

ii) Soit $\varepsilon$ positif et soit $p$ un entier assez grand pour que si les coordonnées de $x$ et $x^{\prime}$ coïncident entre $-p$ et $p,\left|\phi_{n}^{\prime} 1_{\alpha}(x)-\phi_{n}^{\prime} 1_{\alpha}\left(x^{\prime}\right)\right|$ est plus petit que $\varepsilon$. Soit: encore $n_{k}$ une sous-suite de $N$ telle que les suites $\phi_{n_{k}}^{\prime} 1_{\alpha}$ et $\phi_{n_{k}-p}^{\prime} 1_{\alpha}$ convergent uniformément quand $k$ tend vers l'infini vers respectivement $h_{1}$ et $h_{2}$. On a $\inf _{x} h_{1}=\lim _{k} \inf _{x} \phi_{n_{k}}^{\prime} 1_{\alpha}=m\left(1_{\alpha}\right), \inf _{x} h_{2}=m\left(1_{\alpha}\right)$ et $\sup _{x} h_{2}=M\left(1_{\alpha}\right)$. Soit enfin $x$ un point où $h_{1}$ atteint son minimum - On a:

$$
\phi_{n_{k}}^{\prime} 1_{\alpha}(x)=\frac{\sum_{x^{\prime} \in p(x)} \varrho_{n_{k} ; n_{k}-p}(x !) \phi_{n_{k}-p}^{\prime} 1_{\alpha}\left(x^{\prime}\right)}{\sum_{x^{\prime} \in p(x)} \varrho_{n_{k}, n_{k}-p}\left(x^{\prime}\right)} .
$$

iii) On peut voir de plus que les coefficients

$$
\frac{\varrho_{n_{k}, n_{k}-p}\left(x^{\prime}\right)}{\sum_{x^{\prime} \in p(x)} \varrho_{n_{k}, n_{k}-p}\left(x^{\prime}\right)} \quad \text { sont minorés uniformément par un nombre } a_{p} \text {. }
$$

En effet, d'après la proposition 4 les $\varrho_{n_{k}, n_{k}-p}\left(x^{\prime}\right)$ sont de la forme:

$$
\exp -\left(U(\beta)+W\left(\beta, \widehat{\left.y_{n_{k}}(\hat{x})\right)}\right) \sum_{\hat{x} \in\left(n_{k}-p\right)\left(x^{\prime}\right)} f\left(\alpha_{n_{k}-p}(\hat{x}), y_{n_{k}-p}(\hat{x})\right) .\right.
$$

L'exponentielle est comprise entre $e^{-2 p\|\Phi\|}$ et $e^{2 p\|\Phi\|}$ pour tout $x^{\prime}$ de $p(x)$; les termes de la somme, pour deux $x^{\prime}$ différents sont un à un dans un rapport compris entre $e^{-\|\Phi\|_{1}}$ et $e^{\|\Phi\|_{1}}$. D'où on peut calculer $a_{p}$.

iv) Alors quand $n_{k}$ tend vers l'infini, l'équation ii) montre que $h_{1}(x)$ est un point du segment des valeurs prises par $\sum_{x^{\prime} \in p(x)} a_{x^{\prime}} h_{2}\left(x^{\prime}\right)$, avec 
$a_{x^{\prime}} \geqq a_{p}>0$ et $\Sigma a_{x^{\prime}}=1$. Comme $h_{1}(x) \leqq h_{2}\left(x^{\prime}\right)$ pour tout $x^{\prime}$, ce n'est possible que si $h_{2}\left(x^{\prime}\right)=h_{1}(x)=m\left(1_{\alpha}\right)$, pour tout $x^{\prime}$ dans $p(x)$.

v) Mais pour tout point $y$ de $K$, il existe un $x^{\prime}(y)$ dans $p(x)$ dont les coordonnées entre $-p$ et $p$ coïncident avec celles de $y$. On a donc:

$$
M\left(1_{\alpha}\right)-m\left(1_{\alpha}\right)=\sup _{y} h_{2}(y)-m\left(1_{\alpha}\right)=\sup _{y} h_{2}(y)-h_{2}(x(y)),
$$

ce qui est plus petit que $\varepsilon$ d'après le choix de $p . M\left(1_{\alpha}\right)-m\left(1_{\alpha}\right)$ est inférieur à $\varepsilon$, pour tout $\varepsilon$. C.Q.F.D.

D'après le paragraphe 2 , si $\Phi \in B_{1}$, le système $(K, \mu ; T)$ est un $K$-système; on va voir que c'est en fait un schéma de Bernoulli.

On va encore utiliser la construction de la mesure $\mu$ pour montrer:

Proposition 6. Soit $\mu$ la mesure d'équilibre pour $\Phi \in B_{1}$, et soit $v$ la mesure définie par $v(A \times B)=\mu(A) \mu(B)$, si $A \in P_{-\infty, 0]}$ sur les pavés de $P_{(-\infty, 0]} \otimes P_{[1,+\infty)}$ et prolongée ensuite à $\mathscr{A} . \mu$ et $v$ sont équivalentes.

Démonstration. On applique à $v$ les opérateurs $\phi_{n}^{\prime *}$; la propriété résulte de deux lemmes:

Lemma 1. $\phi_{n}^{\prime *} v$ est équivalente à $v$ et $\frac{d \phi_{n}^{*} v}{d v}$ est donné par:

$$
\frac{d \phi_{n}^{\prime *} v}{d v}(x)=\frac{f\left(\alpha_{n}(x), y_{n}(x)\right)}{\sum_{\alpha_{n} \in P_{1-n, n]}} f\left(\alpha_{n}, y_{n}(x)\right) \times E_{v}^{P_{(\infty,-n] \cup] n,+\infty)}} 1_{\alpha_{n}(x)}\left(y_{n(x)}\right)} v \text { p.s. }
$$

Il suffit de le vérifier pour $\phi_{1}^{\prime}$, les autres vérifications sont tout à fait analogues:

Soit $Q v$ le mesure définie par (cf. [3]):

$$
\begin{gathered}
Q v\left(\left\{x ; x_{-m} \in \alpha_{-m}, \ldots, x_{0} \in \alpha_{0} ; x_{1} \in \beta_{1}, \ldots, x_{m} \in \beta_{m}\right\}\right) \\
=v\left(\left\{x ; x_{-m+1} \in \alpha_{-m}, \ldots, x_{0} \in \alpha_{-1} ; x_{1} \in \beta_{2}, \ldots, x_{m-1} \in \beta_{m}\right\}\right) .
\end{gathered}
$$

On a $\phi_{1}^{\prime *} v=\frac{f\left(\alpha_{1}(x), y_{1}(x)\right)}{\sum_{\alpha \in P_{[0,1]}} f\left(\alpha, y_{1}(x)\right)} Q v$.

De même vérifier que

$$
\frac{d v}{d Q v}=g(x)=E^{P_{(-\infty, 1] \cup] 1,+\infty)}} 1_{\alpha_{1}}(x)\left(y_{1}(x)\right)
$$

revient à vérifier que

Or

$$
\phi_{g}^{*} v=v, \quad \text { où } \quad \phi_{g} h(x)=\sum_{x^{\prime} \in 1(x)} g\left(x^{\prime}\right) h\left(x^{\prime}\right) .
$$

$$
\begin{aligned}
\int \phi_{g} h d v & =\int \sum_{x^{\prime} \in \mathcal{I}_{(x)}} E_{v}^{P_{[0,1]^{c}}} 1_{\alpha_{1}\left(x^{\prime}\right)}\left(y_{1}\left(x^{\prime}\right)\right)\left(h\left(x^{\prime}\right)\right) d v(x) \\
& =\int E_{v}^{P_{0}(]^{\mathrm{c}}} h\left(y_{1}\left(x^{\prime}\right)\right) d v(x) .
\end{aligned}
$$


Mais $\alpha y_{1}\left(x^{\prime}\right)$ est un point tel que $S\left(\alpha y_{1}\left(x^{\prime}\right)\right)=x$, si $S$ désigne la transformation

$$
S\left(\ldots x_{-n}, \ldots, x_{0} ; x_{1} \ldots x_{n} \ldots\right)=\left(\ldots x_{-n-1}, \ldots, x_{-1} ; x_{2}, \ldots, x_{n+1} \ldots\right) .
$$

On a donc

$$
\int \phi_{g} h d v=\int E_{v}^{P^{[0,1]^{c}} h\left(S^{-1} x\right) d v(x) .}
$$

Comme $v$ est invariante par $S$, on a finalement:

$$
\int \phi_{g} h d v=\int E_{v}^{P_{[0,1]^{c}}} h d v=\int h d v \text {. }
$$

En écrivant le rapport $\frac{d \phi_{1}^{\prime *} v}{d v}=\frac{d \phi_{1}^{\prime *} v}{d Q v} \times \frac{d Q v}{d v}$, on obtient la formule annoncée.

Soit $\omega$ un point de $K$; dans le suite on écrira $\alpha(\beta)$ pour leś premières coordonnées négatives (positives), $x(y)$ pour les suivantes. Par example, pour la fonction $g$ :

$$
g(\omega)=E_{\mu}^{P_{(-\infty, \text { o[ }}} 1_{\alpha_{0}}(x) \times E_{\mu}^{P_{11,+\infty)}} 1_{\beta_{1}}(y) .
$$

Lemme 2. Les fonctions $\frac{d \phi_{n}^{*} v}{d v}, n \in N$, sont bornées inférieurement et supérieurement.

Démonstration. Il suffit de calculer $\frac{d \phi_{n}^{* *} v}{d v}$ en se permettant des approximations uniformes pour $W(x, y)$ :

vaut, à $e^{6 k_{n}}$ près:

$$
\frac{\exp -(U(\alpha \beta)+W(\alpha \beta, x y))}{\sum_{\alpha^{\prime}, \beta^{\prime}} \exp -\left(U\left(\alpha^{\prime} \beta^{\prime}\right)+W\left(\alpha^{\prime} \beta^{\prime}, x y\right)\right)}
$$

$$
\frac{\exp -(U(\alpha)+U(\beta)+W(\alpha, \beta)+W(\alpha, x)+W(\beta, y))}{\sum_{\alpha^{\prime}, \beta^{\prime}} \exp -\left(U\left(\alpha^{\prime}\right)+U\left(\beta^{\prime}\right)+W\left(\alpha^{\prime}, \beta^{\prime}\right)+W\left(\alpha^{\prime}, x\right)+W\left(\beta^{\prime}, y\right)\right)}
$$

on le majore par:

$$
e^{2\|\Phi\|_{1}} \frac{\exp -(U(\alpha)+W(\alpha, x)) \times \exp -(U(\beta)+W(\beta, y))}{\sum_{\alpha^{\prime}, \beta^{\prime}} \exp -\left(U\left(\alpha^{\prime}\right)+W\left(\alpha^{\prime}, x\right)+U\left(\beta^{\prime}\right)+W\left(\beta^{\prime}, y\right)\right)} .
$$

On va estimer de même

$$
\begin{gathered}
E^{\left.P_{-\infty},-n\right]} 1_{\alpha}(x)=E^{P_{(-\infty,-n]}}\left(E^{P_{(-\infty,-n] \cup[1,+\infty)}} 1 \alpha\left(x y^{\prime \prime}\right)\right)(x) \\
E^{P_{(-\infty,-n] \cup[1,+\infty)}} 1_{\alpha}\left(x y^{\prime \prime}\right)=\frac{\exp -\left(U(\alpha)+W\left(\alpha, x y^{\prime \prime}\right)\right)}{\sum_{\alpha^{\prime}} \exp -\left(U\left(\alpha^{\prime}\right)+W\left(\alpha^{\prime}, x y^{\prime \prime}\right)\right)}
\end{gathered}
$$


ce qui vaut, à $e^{4 k_{n}}$ près,

$$
\frac{\exp -\left(U(\alpha)+W(\alpha, x)+W\left(\alpha, y^{\prime \prime}\right)\right)}{\sum_{\alpha^{\prime}} \exp -\left(U\left(\alpha^{\prime}\right)+W\left(\alpha^{\prime}, x\right)+W\left(\alpha^{\prime}, y^{\prime \prime}\right)\right)}
$$

que l'on peut minorer par:

$$
e^{-2\|\Phi\|_{1}} \frac{\exp -(U(\alpha)+W(\alpha, x))}{\sum_{\alpha^{\prime}} \exp -\left(U\left(\alpha^{\prime}\right)+W\left(\alpha^{\prime}, x\right)\right)}
$$

qui est une fonction $P_{(-\mathrm{p},-n]}$ mesurable. En regroupant dans la formule du lemme 1 , tout se simplifie et il reste, à $e^{14 k_{n}}$ près: $\frac{d \phi_{n}^{\prime *} v}{d v} \leqq e^{6\|\Phi\|_{1}}$. On aurait de même $\frac{d \phi_{n}^{\prime} v}{v} \geqq e^{-6\|\Phi\|_{1}}$.

On peut maintenant achever la démonstration de la proposition 6; en effet les lemmes 1 et 2 assurent que les fonctions $\frac{d \phi_{n}^{* \prime} v}{d v}$ forment une famille relativement compacte pour la topologie $\sigma\left(L^{1}(v), L^{\infty}\right)$, et que si $f$ est un point d'adhérence de cette famille, $f$ est strictement positive $v$ presque-sûrement. Mais alors $f v$ est un point d'adhérence faible de la famille de mesures $\phi_{n}^{*} v$; c'est donc la mesure d'équilibre $\mu$ (propositions 1 et 5). $\mu$ est bien équivalente à $v$. On peut maintenant montrer:

Théorème. Si $\Phi \in B_{1}$, le système $(K, \mu ; T)$, où $\mu$ est la mesure d'équilibre pour $\Phi$ est un schéma de Bernoulli.

Démonstration. La partition $P_{0}$ a en effet une propriété de Bernoulli faible qui est suffisante pour engendrer un schéma de Bernoulli (W. B. cf. [2]):

- Pour tout $\varepsilon$, il existe $n$ tel que $P_{[-l, 0]}$ soit $\varepsilon$-indépendante de $P_{[n, m]}$, pour tous les entiers $l$ et $m$.,$- l^{\prime} \varepsilon$-indépendance de deux $\sigma$-algèbres atomiques étant définie par:

$\sum_{p q}|\mu(p q)-\mu(p), \mu(q)|<\varepsilon$, où $p$ et $q$ décrivent les atomes des deux algèbres.

Avec les notations de la proposition précédente, la propriété est équivalente à $[\mu-v]_{P_{(-\infty,-n] \cup[n,+\infty)}}$ tend vers zéro quand $n$ tend vers l'infini, où $[m]_{\mathscr{A}}$ désigne la variation totale de la mesure $m$ restreinte à l'algèbre $\mathscr{A}$. C'est encore équivalent à $\left\langle\mu=v\right.$ sur $\left.\bigwedge_{n} P_{[-n, n]^{c}}\right\rangle$ par continuité. C'est enfin vrai pour $P_{0}$, car $\mu$ est triviale sur $\bigwedge_{n} P_{[-n, n]^{c}}$ (propositions 3 et 5), et $v$, qui lui est équivalente ne peut que coïncider avec $\mu \operatorname{sur} \bigwedge_{n} P_{[-n, n]^{c}}$. 


\title{
Bibliographie
}

1. Conze, J.P.: Entropie d'un groupe abélien de transformations. Z. Wahrscheinlichkeitstheorie verw. Geb. 25, 11 (1972)

2. Friedman, N., Ornstein, D.S.: An isomorphism of weak Bernoulli transformations. Advances in Math. 1970, 5

3. Keane, M.: Strongly mixing $g$-measures. Inventiones math. 16, 309 (1972)

4. Ruelle, D.: Statistical mechanics. Rigorous results. New York: Benjamin 1969

5. Ruelle, D.: Statistical mechanics of a one-dimensional lattice gas. Commun. math. Phys. 9 (1968)

J'ai appris, en rédigeant ce travail que le théorème est montré dans

6. Gallavotti, G.: Commun. math. Phys. 32, 183-190 (1973)

\author{
F. Ledrappier \\ Laboratoire de Probabilités T 56 \\ 9 Quai Saint-Bernard \\ Paris 5, France
}

